\title{
Sarcomatoid Carcinoma of the Lung Mimicking a Thromboembolic Disease
}

\author{
Mona Mlika, ${ }^{1,2}$ Selma Chaieb, ${ }^{1,2}$ Sonia Baccari, ${ }^{3}$ Tarek Kilani, ${ }^{3}$ Faouzi Mezni1,2 \\ 1. Department of Pathology, Abderrahman Mami Hospital, Université de Médecine, Tunis El Manar; 2. Research Unit: RU12/SP16, Public \\ Health Ministry, Tunis, Tunisia; 3. Department of Thoarcic Surgery, Abderrahman Mami Hospital, Tunis, Tunisia
}

DOl: http://doi.org/10.17925/ERPD.2016.02.02.58

B ackground: Sarcomatoid carcinoma of the lung is a rare tumour with a poor prognosis. Observation: We present a case report of sarcomatoid carcinoma and its mode of presentation with a complete illustration. In this case, the tumour, which is generally peripheral, was proximal and was revealed by non-specific symptoms and radiological features suggesting a thromboembolic disease. Conclusion: This observation is interesting because it highlights the necessity of evoking the diagnosis of lung carcinoma when dealing with radiologic features related to a thromboembolic disease.

\section{Keywords}

Sarcomatoid carcinoma, thromboembolic disease, pathology.

Disclosure: Mona Mlika, Selma Chaieb, Sonia Baccari, Tarek Kilani and Faouzi Mezni have nothing to disclose in relation to this article. No funding was received for the publication of this article.

Compliance with Ethics: All procedures were followed in accordance with the responsible committee on human experimentation and with the Helsinki Declaration of 1975 and subsequent revisions, and informed consent was received from the patient involved in this case study. open Access: This article is published under the Creative Commons Attribution Noncommercial License, which permits any non-commercial use, distribution, adaptation and reproduction provided the original author(s) and source are given appropriate credit.

Received: 11 June 2016

Accepted: 10 August 2016

Citation: European Respiratory \& Pulmonary Diseases, 2016;12(2):58-9

Corresponding Author: Mona Mlika, Department

of Pathology, Abderrahman Mami Hospital,

Université de Médecine, Tunis El Manar.

E:mlika.zorgati.mona@hotmail.com
Primary lung carcinoma spreading to pulmonary vessels and presenting as a thromboembolic disease is quite rare and only few individual cases have been reported in the English literature. . $^{1-3}$ The prognostic significance of pulmonary vessel invasion by lung carcinoma is unclear and the treatment is challenging. We aim to describe an unusual case of sarcomatoid carcinoma of the lung characterised by its hilar localization. ${ }^{1,2}$

\section{Case report}

A 68-year-old man, 80 pack-year smoker, consulted for a 4-month-lasting effort dyspnea of New York Heart Association (NYHA) functional class II and dry cough. Physical examination revealed a grade III/IV ejection systolic murmur in the aortic area. Chest X-ray showed a heterogeneous poorly defined opacity of the right hilius without cardiomegaly (Figure 1). Electrocardiogram showed a regular sinusoidal rhythm and a tachycardia at 100 beats/min without signs of left ventricular hypertrophy. A two-dimensional (2D) transthoracic echocardiography (TTE) showed a severe aortic stenosis. The left ventricular ejection fraction was estimated to $70 \%$. There was no pulmonary arterial hypertension. The pulmonary artery systolic pressure was estimated to $30 \mathrm{~mm} \mathrm{Hg}$. A computed tomography (CT) scan revealed a thrombus in the lower branch of the right pulmonary artery of tumoural appearance extending to the right lower lobe artery (Figure 2). Bronchial fibroscopy was normal. No distant metastasis was revealed by the cerebral CT scan or the abdominal CT scan. The lung function was within normal values. Surgical resection of the arterial process and aortic replacement was decided.

Surgical resection was performed through a median vertical sternotomy. The procedure consisted of a right intra pericardial pneumonectomy with aortic valve replacement by a mechanical valve. A mediastinal lymph node dissection was also performed. Postoperative macroscopic examination of the resected specimen showed a $2 \mathrm{~cm}$ tumour encompassing the pulmonary artery and developing into the sub-intima without endoluminal component (Figure 3A). The extemporaneous exam concluded to a sarcomatous tumour. Definitive microscopic findings consisted in a malignant proliferation with wide necrosis composed of tumour cells of variable size with abundant eosinophilic cytoplasm and atypical nuclei. Some tumour cells were multinucleated (Figure 3B). Immunohistochemical study revealed the co-expression of cytokeratin and vimentin antibodies by the tumour cells (Figure $3 C$ and $D$ ). Other antibodies including anti-smooth muscle actin and desmin weren't expressed, ruling out the diagnosis of a muscle differentiated tumour. The mediastinal lymph nodes dissected were benign.

These morphological and immunohistochemical features were consistent with a primary sarcomatoid carcinoma of the lung invading the pulmonary artery classified as a pT1NOMO tumour. Molecular studies highlighted no epidermal growth factor receptor (EGFR), Her mutations, anaplastic lymphoma kinase (ALK) translocation or c-met amplification.

There were no postoperative serious complications except for an atelectasis with oxygen dependency. This complication was managed by a non-invasive ventilation and chest physiotherapy with a good outcome. The patient was discharged 18 days after the surgical resection. He is still 
Figure 1: Chest X-ray showing a heterogeneous poorly defined opacity projecting over the right hilius on posterioranterior view, without cardiomegaly

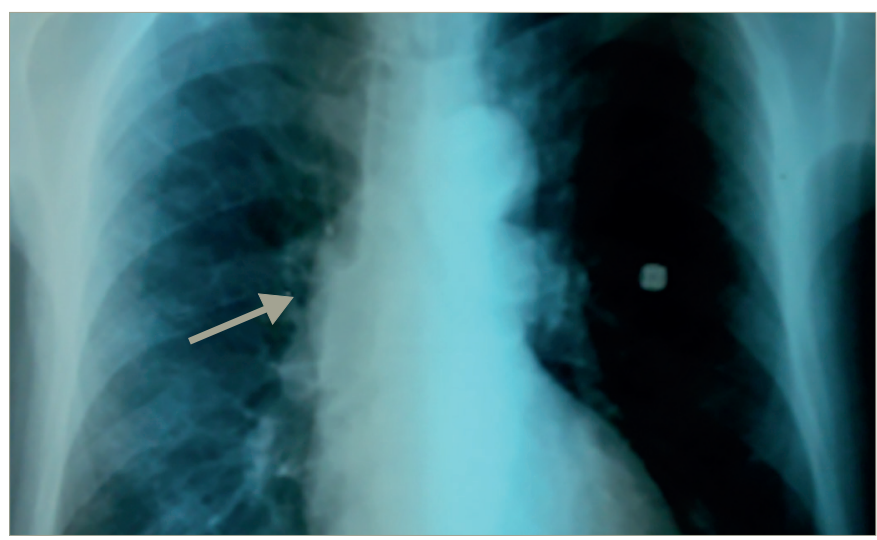

followed by the oncologist, and after 4 months of follow-up, presented no complications.

\section{Discussion}

This case was interesting because it illustrated a rare presentation of sarcomatoid carcinoma of the lung. This tumour is very rare and mainly peripherally localised. The hilar localisation induced nonspecific presenting symptoms, which were confused with pulmonary thromboembolism. ${ }^{3}$ The artery obstruction may be caused by an intrinsic or extrinsic tumour. ${ }^{4}$ In the case reported here, the tumour development was sub-intimal without an intra luminal development. It was revealed by the $\mathrm{CT}$, which represents the standard reference method for the diagnosis of acute pulmonary thromboembolism and was also reported by some authors to have prognostic value. ${ }^{5}$ Sarcomatoid carcinoma of the lung accounts for 0.3 to $0.1 \%$ of all lung malignancies. It was recognised as a distinct entity by the World Health Organization classification (WHO 1999), updated in 2004 and 2015. ${ }^{1}$ It is defined as a poorly differentiated non-small cell carcinoma that contains sarcomatous component or sarcoma-like elements. It represents an overall continuum of epithelial and mesenchymal differentiation. ${ }^{1,6}$ The diagnosis is based on the microscopic and the immunohistochemical examination. Immunohistochemical analysis shows cytokeratin positivity (most often AE1AE3, CAM5.2, CK18, CK7). Vimentin, carcinoembryonic antigen (CEA) and smooth muscle markers can also be positive, but positivity of epithelial markers is not essential if components of conventional cancer are morphologically present. ${ }^{6}$

Surgical resection is the mainstay treatment. Adjuvant therapy is sometimes used but is poorly effective. ${ }^{1,6}$ The prognosis is worse than the prognosis of other non-small cell lung carcinomas. ${ }^{1}$ It essentially depends on the size and extent of the tumour. Some studies suggest that blood vessel invasion is a major prognostic factor associated with shorter survival. $3,7,8$ In this case, the tumour had invaded the pulmonary artery. The rarity of this tumour growth pattern makes it difficult to diagnose. The blood invasion observed in sarcomatoid carcinoma is thought to lead to dissemination of the tumour in the pulmonary
Figure 2: Computed tomography scan showing a polypoid lesion situated in the right pulmonary artery (arrow)

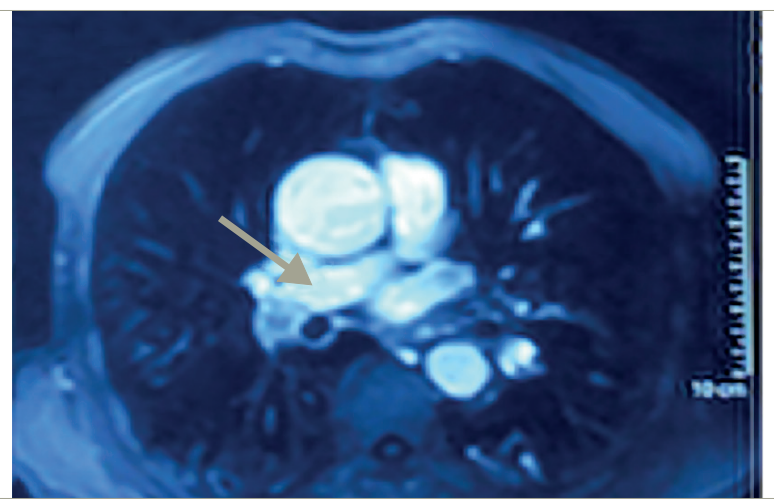

Figure 3: Results of tumour lobectomy and immunohistochemical analysis

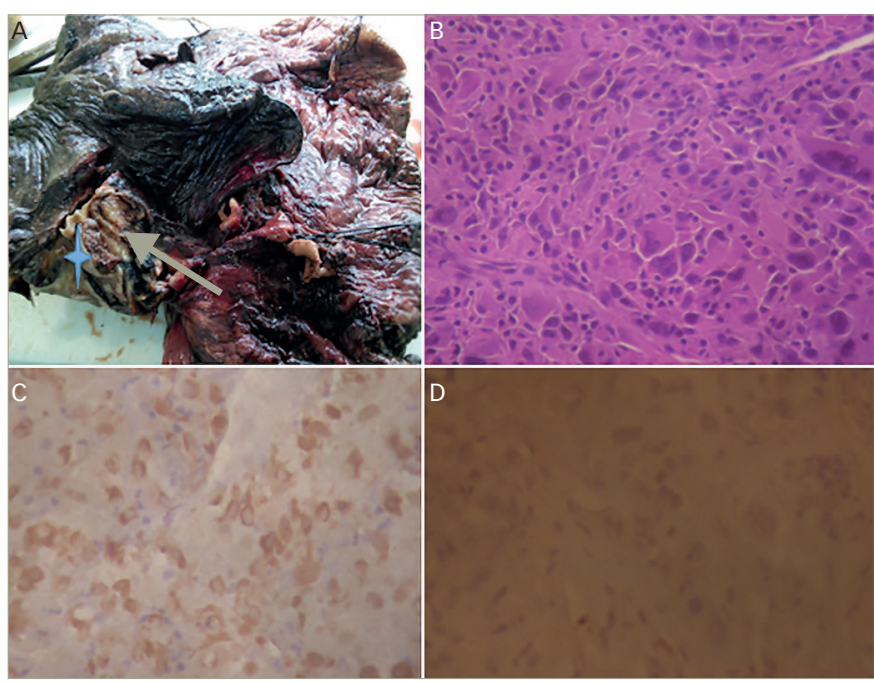

A. Gross findings of a lobectomy showing a tumour (arrow) developed beyond the intimal lining of the pulmonary artery (star). B. Microscopic examination showing a diffuse malignant tumour made of polymorphic cells with atypical nuclei (HES x 400). C. Immunohistochemical findings showing the expression of the cytokeratin and the vimentin antibodies by the tumour cells (HES x 400). D. immunohistochemical study showing the negativity of the tumour cells with the TTF1, P40, myogenin and desmin antibodies (HES X 400).

circulation, resulting in multiple intrapulmonary metastases. ${ }^{8}$ However, in our case, such pathological findings were not observed.Thus, even when macroscopic tumour invasion to the pulmonary artery is observed, complete surgical resection of the tumour may offer a better prognosis. ${ }^{9}$

\section{Conclusion}

We have presented a case of lung sarcomatoid carcinoma invading the pulmonary artery. This mode of tumour extension is rare and its prognostic significance remains unclear, especially in such a subtype. We aimed to highlight that pulmonary artery invasion by a lung carcinoma is part of the differential diagnosis of chronic thromboembolic disease. Although blood vessel invasion is generally an ominous prognostic factor, curative surgical resection should improve the prognosis. $\square$
Roberts TE, Hasleton PS, Musgrove $C$, et al. Vascular invasion in non-small cell lung carcinoma, J Clin Pathol, 1992:45:591-3.

Kharwar RB, Sharma A, Sethi R, et al., Pulmonary Artery Stenosis due to Lung Carcinoma: A Rare Cause of Dyspnea, J Cardiovasc Ultrasound, 2014;22:209-12.

3. Millet $A$, Morel $H$, Sanchez $O$, et al., Invasion of the pulmonary artery by an undifferentiated carcinoma, Rev Mal Respir, 2008;25:63-7.

4. Jaffery Z1, Ananthasubramaniam K, Isolated left pulmonary artery stenosis due to extrinsic compression by intra thoracic tumor recognition of unusual Doppler flow pattern and correlation with computed tomography, Int J Cardiovasc Imaging, 2007:23:507-10 Plasencia-Martínez JM, Carmona-Bayonas A, Calvo-Temprano D, Jiménez-Fonseca P, Prognostic value of computed tomography in acute pulmonary thromboembolism [article in Spanish], Radiologia, 2016;1: Online first. DOl: 10.1016/j.rx.2016.06.011.

6. Szkorupa M, Bohanes T, Neoral C, et al., Sarcomatoid carcinoma of the lung. A case report, Klin Onkol, 2015;28:57-60.
Raveglia F Mezzetti M, Panigalli T, et al, Personal experience in surgical management of pulmonary pleomorphic carcinoma Ann Thorac Surg 2004:78:1742-7.

8. Vieira T, Antoine M, Ruppert AM, et al., Blood vessel invasion is a major feature and a factor of poor prognosis in sarcomatoid carcinoma of the lung, Lung Cancer, 2014;85:276-81.

Yamaguchi T, Suzuki K, Asamura H, et al., Lung carcinoma with polypoid growth in the main pulmonary artery: report of two cases, Jpn J Clin Oncol, 2000;30:358-61. 